\title{
Intentional binding and higher order agency experience
}

James W. Moore $^{1 *} \&$ Patrick Haggard ${ }^{2}$

${ }^{1}$ Department of Psychiatry, Brain Mapping Unit, University of Cambridge, Downing Site, Cambridge, CB2 3EB, UK

${ }^{2}$ Institute of Cognitive Neuroscience, University College London, London, WC1N 3AR, UK

*Corresponding Author: jm697@ cam.ac.uk

\begin{abstract}
Recent research has shown that human instrumental action is associated with systematic changes in time perception: The interval between a voluntary action and an outcome is perceived as shorter than the interval between a physically similar involuntary movement and an outcome. The study by Ebert and Wegner (this issue) suggests that this change in time perception is related to higher order agency experience. Notwithstanding certain issues arising from their study, which are discussed, we believe it offers validation of binding as a measure of sense of agency.
\end{abstract}

Keywords: Sense of agency; Intentional binding; Voluntary action; Consciousness; Volition In press, Consciousness and Cognition (2010) - Uncorrected proof 


\section{Main text}

Recent research has shown that human instrumental action is associated with systematic changes in time perception: The interval between a voluntary action and an outcome is perceived as shorter than the interval between a physically similar involuntary movement and an outcome (Haggard, Clark, \& Kalogeras, 2002; Engbert, Wohlschläger, \& Haggard, 2008; Moore, Wegner, \& Haggard, 2009). This has been referred to as 'intentional binding'. These modulations in time perception may be related to the feeling of controlling actions. For example, if binding shortens the perceived interval between action and effect, it may enhance the sense of agency, because temporal contiguity plays an important role in both causation (Hume, 1739) and sense of control (Wegner, 2002). However, the binding measure is indirect, and does not explicitly measure the sense of agency.

Ebert \& Wegner's study (this issue) is, to our knowledge, the first attempt to link time perception to an explicit agency measure. In particular, they focus on the feeling of authorship, which they define as an actor 'perceiving his or her action as the cause of the subsequent event'. In two experiments they found that manipulating the consistency of the relation between a participant's action and its effect modulated both perceived interval length and explicit judgements of authorship. Interestingly, when the two measures were taken on each trial, there was a significant correlation between them. However, when timing judgements and authorship judgements were made in separate blocks of trials, there was no significant correlation.

Whilst we find the data reported by these authors very clear, and their conclusions interesting, we think their article draws attention to some important issues: 
1) It is unclear whether the effect of consistency is driven by predictive or retrospective processes. Both are likely to contribute to sense of agency (Moore \& Haggard, 2008). The authors themselves suggest that prediction plays a role: 'the effect of consistency was driven by participants' prior expectations about how objects should react to pushing and pulling'. But they also suggest that the effects of consistency 'cannot be determined until after an event occurs', implying a role for retrospective influences. Consistency affects binding and agency judgements in similar ways, but does not clearly distinguish between the predictive and the retrospective processes underlying these judgements.

2) The causal relation between binding and authorship is unclear. Do people feel agency because they perceive actions and effects as closely linked in time, or do they have a contracted experience of action-effect interval because they feel they are in control? Alternatively, binding and authorship could both be independent effects of some other factor. In the experiments reported, the order of judgements was constant. Timing judgements were always performed first and explicit agency judgements second. This might promote the influence of intentional binding on self reports (but see point 3 below). To distinguish between these possibilities would require an interventional experimental design, in which an experimental factor independently manipulated either binding or explicit authorship, and the effects on the other measure were assessed.

3) Ebert and Wegner found that testing intentional binding and self-reports in separate blocks eliminated the correlation between measures. This finding recalls studies of human learning, in which implicit and explicit measures of learning may 
'contaminate' each other when tested on the same trial (Perruchet, Cleeremans, \& Destrebecqz, 2006). Interestingly, such studies show that this contamination is reduced when the two measures are obtained in separate blocks of trials.

The results of Ebert and Wegner's second experiment may therefore represent the effects of consistency on binding and self-reports more purely. The data in Experiment 2 show that consistency has a stronger effect on self-reports than binding. This would be expected as binding is known to occur for arbitrary consequences of action, such as tones (Haggard et al., 2002). However, when self-reports and binding measures were taken on each trial, as in Experiment 1 the effect of consistency on binding was stronger. This suggests that explicitly reporting the feeling of authorship on each trial may have increased the effects of consistency on binding. This suggests that explicit decisions about authorship may contribute to binding. Nevertheless, a more conclusive demonstration of this might come from independently varying some factor that influences the sense of authorship, and assessing effects on binding.

4) Ebert and Wegner show that binding is more sensitive to consistency at shorter delays, whereas authorship judgements showed no such effect of delay. This is what we would expect based on early studies on binding which showed that longer actionoutcome intervals were associated with reduced binding (Haggard et al., 2002). This work implied that the binding effect is restricted to sensorimotor timescales. However, more recent work by Buehner and Humphreys (2009) has shown binding for much longer intervals. For the moment, the temporal range in which binding operates remains unclear. 
We welcome Ebert and Wegner's study for a number of reasons. First, they replicate the basic binding effect, and confirm that temporal contiguity is an important factor (cf. Haggard, et al., 2002). Second, their study again demonstrates effects of action on time perception, using a task that does not rely on the much-criticised 'Libet clock' method of reporting event times (see also, Engbert et al., 2008; Moore et al., 2009, for example). Third, the study validates the use of intentional binding as a measure of sense of agency, by providing evidence that quantitative changes in binding are associated with quantitative changes in explicit reports of agency. However, Ebert and Wegner's study does not yet show the mechanism underlying either binding or sense of authorship. That would be the next research step. 


\section{References}

Buehner, M. J., \& Humphreys, G. R. (2009). Causal binding of actions to their effects. Psychological Science: A Journal of the American Psychological Society / APS, 20(10), 1221-1228. doi: 10.1111/j.1467-9280.2009.02435.x

Engbert, K., Wohlschläger, A., \& Haggard, P. (2008). Who is causing what? The sense of agency is relational and efferent-triggered. Cognition, 107(2), 693-704. doi: 10.1016/j.cognition.2007.07.021

Haggard, P., Clark, S., \& Kalogeras, J. (2002). Voluntary action and conscious awareness. Nature Neuroscience, 5(4), 382-5. doi: 10.1038/nn827

Hume, D. (1739). A Treatise of Human Nature. Oxford Oxford University Press.

Moore, J., \& Haggard, P. (2008). Awareness of action: Inference and prediction. Consciousness and Cognition, 17(1), 136-44. doi: 10.1016/j.concog.2006.12.004

Moore, J. W., Wegner, D. M., \& Haggard, P. (2009). Modulating the sense of agency with external cues. Consciousness and Cognition. doi: 10.1016/j.concog.2009.05.004

Perruchet, P., Cleeremans, A., \& Destrebecqz, A. (2006). Dissociating the effects of automatic activation and explicit expectancy on reaction times in a simple associative learning task. Journal of Experimental Psychology. Learning, Memory, and Cognition, 32(5), 955-965. doi: 10.1037/0278-7393.32.5.955

Wegner, D. M. (2002). The Illusion of Conscious Will (illustrated edition.). MIT Press. 